\title{
BMJ Open School-based, two-arm, parallel, controlled trial of a culturally adapted resilience intervention to improve adolescent mental health in Vietnam: study protocol
}

Thach Tran (D) , ${ }^{1}$ Huong Thanh Nguyen, ${ }^{2}$ lan Shochet (D) , ${ }^{3}$ Astrid Wurfl, ${ }^{3}$ Jayne Orr, ${ }^{3}$ Nga Nguyen, ${ }^{2}$ Nga La, ${ }^{2}$ Hau Nguyen, ${ }^{1}$ Ruby Stocker, ${ }^{1}$ Trang Nguyen, ${ }^{1}$ Minh Le, ${ }^{1}$ Jane Fisher (i) ${ }^{1}$

To cite: Tran T, Nguyen HT, Shochet I, et al. Schoolbased, two-arm, parallel, controlled trial of a culturally adapted resilience intervention to improve adolescent mental health in Vietnam: study protocol. BMJ Open 2020;10:e039343. doi:10.1136/ bmjopen-2020-039343

- Prepublication history for this paper is available online. To view these files, please visit the journal online (http://dx.doi. org/10.1136/bmjopen-2020039343).

\section{Received 12 April 2020}

Revised 30 July 2020

Accepted 21 August 2020

\section{Check for updates}

(C) Author(s) (or their employer(s)) 2020. Re-use permitted under CC BY-NC. No commercial re-use. See rights and permissions. Published by BMJ.

${ }^{1} \mathrm{Global}$ and Women's Health, Public Health and Preventive Medicine, Monash University, Melbourne, Victoria, Australia ${ }^{2}$ Department of Health Education, Faculty of Social Science, Behavior and Health Education, Hanoi University of Public Health, Hanoi, Vietnam ${ }^{3}$ School of Psychology and Counselling, Queensland University of Technology, Brisbane, Queensland, Australia

Correspondence to Professor Jane Fisher; jane.fisher@monash.edu

\section{ABSTRACT}

Introduction The Resourceful Adolescent Program (RAP) is an evidence-based resilience intervention for adolescents. Operating in a strength-focused paradigm, the programme uses an integration of cognitive behavioural therapy and interpersonal psychotherapy to improve coping skills and build resilience. This study aims to establish whether a culturally and linguistically adapted intervention informed by RAP principles is effective in increasing resilience, enhancing coping skills and preventing symptoms of depression and anxiety. Methods and analysis We will translate, back-translate and culturally adapt the RAP for adolescents and training materials for facilitators, and the adapted intervention will be called Happy House. A two-arm parallel controlled trial will be conducted in eight high schools in the north of Vietnam. In each of the selected schools, all students from four randomly selected grade 10 classes (an estimation of about 1204 students) will be invited to participate. The control group will receive the usual curriculum. The intervention group will receive sixweekly 90 min school-based group sessions of Happy House in addition to the usual curriculum. The primary outcome, depressive symptoms, will be measured using a locally validated version of the Centre for Epidemiologic Studies Depression Scale Revised. Secondary outcomes are mental well-being, coping self-efficacy, school connectedness, anger management and health risk behaviours. Data will be collected at recruitment, and at two weeks and six months post intervention. Mixed-effect logistic regression for the main outcome and mixed-effect linear and logistic regression models for the secondary outcomes will be conducted to estimate the effects of the intervention on the outcomes.

Ethics and dissemination This trial has been approved by Monash University Human Research Ethics Committee (No. 21455) and the Institutional Review Board of the Hanoi School of Public Health (488/2019/YTCC-HD3). Dissemination of findings will include peer-reviewed publications, international and national conferences, seminar and media presentations, national policy briefings in Vietnam, local language reports and lay language summaries for participants.

\section{Strengths and limitations of this study}

- This will provide evidence for the effectiveness of a sustainable integration of a cultural adaptation of the Resourceful Adolescent Program into the existing school curriculum.

- This is the first trial to address adolescent mental health problems and to promote positive mental health in Vietnam.

- The sample size is large and includes both urban and rural areas.

- Advanced statistical technique is to be used and will allow us to identify the mechanisms of the trial effects.

- This study will not include a sample of out-of-school adolescents.

Trial registration numbers Registered with the Australian New Zealand Clinical Trials Registry, registration number: ACTRN12620000088943 (3/2/2020). WHO Universal Trial Number: U1111-1246-4079.

\section{INTRODUCTION}

Nearly $20 \%$ of the world's population are adolescents, and almost all (85\%) of them live in low and middle-income countries (LMICs). The quality of interactions between adolescents and their families, schools, communities and the broader social and cultural environment influence their development. These interactions are important, as they can both foster healthy development, and contribute to mental health problems. ${ }^{1}$

Adolescents with good mental health are able to develop socially, emotionally and physically, along optimal trajectories, and relate to others at home, school, in the workplace or in social settings. They experience being valued, have increasing competence and autonomy, 
and a hopeful view of the future. Although most adolescents have good mental health, it is also a phase of life when mental health problems can arise, and contribute to morbidity and mortality. ${ }^{12}$ If these problems are unrecognised or neglected, adolescents will have a higher risk of experiencing poor psychological functioning throughout their remaining adolescence and adulthood.

The health and well-being of adolescents is influenced by their experiences of physical and psychological changes and the social environment in which they live. In his ecological model of human development, Bronfenbrenner argued that adolescence is heavily influenced by interactions between the adolescent and their families, peers, adults in positions of authority, and surrounding community, culture and environment. ${ }^{4}$ The quality and quantity of these interactions are strong determinants of developmental progress. The closest determinants are the most powerful; the family, school, local neighbourhood and community. It is important to acknowledge, however, that adolescence occurs in a broader context, where social, cultural, economic and political factors are also influential. This context is dynamic, and reflects globalisation, increasing urbanisation, technology and digital communication and access to information. Many families feel underprepared to assist their children to grow optimally in this changing context.

Mental health problems among adolescents are a major public health concern, and are more prevalent in low and lower-middle-income countries (LALMICs) than high-income countries. ${ }^{56}$ Mental health problems reduce healthy social participation, impede adolescents' development and increase likelihood of psychiatric illness and disability in adulthood. Around $50 \%$ of adult mental health problems originate in childhood and adolescence. $^{2}$ Mental health problems result in significant costs, including lost economic productivity, and increased burden on health, education, social protection and justice systems. ${ }^{78}$

Our review of the evidence available from LMICs about experiences of violence among young people ${ }^{19}$ revealed that rates of mental health problems were higher among particular groups. For example, young people living in fragile situations, such as in civil strife or neighbourhood violence, and experiencing malnutrition, poverty, lack of access to safe accommodation, workplace exploitation, or experiences of sexual, physical and emotional maltreatment were more likely to experience poor mental health. Negative experiences at school, such as bullying, underrecognition of learning difficulties and sensory losses, overambitious expectations of academic achievement and criticism of poor academic performance are associated with higher rates of mental health problems. Similarly, family characteristics varied between adolescents with or without mental health problems; poor household functioning, inadequate caregiving and low emotional attachment, family violence, poor parental mental health and substance abuse were reported more frequently by adolescents with rather than without symptoms. Additionally, mental health problems were more common among adolescent girls in settings where gender discrimination limited opportunities for participation in education; and where blame, shame and guilt associated with female sexuality were the norm. ${ }^{1}$

Resilience is defined as 'a dynamic process encompassing positive adaptation within the context of significant adversity'. ${ }^{10}$ Resilience helps young people overcome the impacts of traumatic events, including exposure to violence. ${ }^{11}$ Interventions to promote the growth of resilience are required for adolescents from LMIC to optimise their mental health, functioning and healthy development. Resilience can be promoted by enhancing protective factors (such as improved coping skills) that moderate or mediate the effects of adversity on mental health. $^{12}$

\section{Mental health problems among adolescents in Vietnam}

We surveyed 1745 students aged $16-18$ years in ten schools in Hanoi ${ }^{13}$ Prior year suicidal thoughts were reported by $21.4 \%(95 \%$ CI $18.5 \%$ to $24.5 \%)$ of the girls and $7.9 \%$ (95\% CI $6.2 \%$ to $9.8 \%)$ of the boys. Prior year suicidal plans were reported by $7.8 \%$ (95\% CI $5.9 \%$ to $9.8 \%$ ) of the girls and $4.0 \%$ (95\% CI $2.7 \%$ to $5.3 \%$ ) of the boys. Exposure to multiple forms of victimisation, including child abuse and neglect, witnessing family or neighbourhood violence, property victimisation and cyber bullying, was prevalent among the students: $94.3 \%$ (95\% CI: $92.5 \%$ to $95.4 \%$ ) had experienced at least one form of victimisation during their lifetime and $31.1 \%$ (95\% CI: $27.8 \%$ to $33.5 \%$ ) had experienced ten or more forms (termed polyvictimisation). ${ }^{14}$ Polyvictimisation was associated with significantly increased symptoms of depression and anxiety, likelihood of health risk behaviours, suicidal behaviours and poorer health-related quality of life among both girls and boys. ${ }^{14} 15$

Rates of victimisation were similar to those found in a survey of 2591 Vietnamese adolescents aged 12-18 years, ${ }^{16}$ in which more than $39 \%$ reported experiences of emotional abuse, $47 \%$ reported experiences of physical abuse, nearly $20 \%$ reported that they had experienced sexual abuse and 29\% reported that they had experienced neglect. The data aligned with those of Akmatov ${ }^{17}$ who found that more than $55 \%$ of adolescents in Vietnam had experienced moderate physical abuse perpetrated by their parents. A cross-sectional study of 1161 students aged 15-19 years examined the burden of mental health problems among secondary school students in Can Tho city, Vietnam. ${ }^{18}$ The prevalence of clinically significant symptoms of depression and anxiety were $41.1 \%$ and $22.8 \%$, respectively.

A responsive and warm parent-adolescent relationship protects against adolescent mental health problems, including depression. ${ }^{12}$ A lack of communication and understanding between parents and adolescents increases the risk of poor adolescent mental health. A recent survey of high school students in Vietnam found poor communication between parents and adolescents had a negative 
impact on young people's mental health, and contributed to low self-esteem, feelings of sadness and loneliness and suicidal thoughts. ${ }^{19}$

Adolescents' sense of belonging and connectedness to their schools play an important role in their mental health. Analyses of data from the Survey Assessment of Vietnamese Youth (SAVY) I and SAVY II showed that adolescents who felt connected to their schools were less likely to report psychological symptoms. ${ }^{20}$

\section{Mental health policies and programs for adolescent mental health}

School-based mental health interventions worldwide, which target the life skills and resilience of adolescents, have resulted in improved self-esteem, motivation and self-efficacy. ${ }^{21} 22$ Parenting programmes that aim to improve parent-child relationships and reduce parentchild conflict and abuse have also been found to be successful. ${ }^{322}$ Interventions to reduce adolescent risktaking behaviours appear to have a beneficial impact on knowledge and attitudes, the precursors to behaviour change. $^{23}$

The Resourceful Adolescent Program (RAP) ${ }^{24}$ developed by Shochet and Wurfl aims to build psychological resilience and promote positive mental health among young people. The programme has been implemented in Australia and other settings, but only a few in LALMIC.

The RAP for adolescents is a school-based programme to promote interpersonal protective factors for adolescent depression and other mental health problems. It integrates elements of cognitive behavioural therapy $(\mathrm{CBT})^{25}$ and interpersonal psychotherapy ${ }^{26}$ which are the most strongly evidence-informed interventions for adolescent depression. ${ }^{27}$ The RAP is brief and efficient, being delivered over $11 \times 45$ min group sessions. The CBT components of the programme promote self-regulation and affect regulation through increasing the capacity to keep calm in the face of stress and adversity, improving cognitive reframing to develop more positive self-talk, and building capacity for problem solving. The interpersonal components of the programme encourage participants to form and maintain support networks and to reduce interpersonal conflict by applying an understanding of others' perspectives. The RAP is a strengthbased programme, where the focus is on identifying and developing strengths rather than on pathology.

The efficacy and effectiveness of RAP have been demonstrated in randomised controlled trials, which have shown that adolescents who received RAP had statistically and clinically significantly lower levels of depressive and hopelessness symptoms post intervention and at 18-month follow-up, than those in control groups. ${ }^{28-30}$ In one trial in Brisbane, Australia, three groups were compared: RAP-A (for adolescents), RAP-F (RAP-A for adolescents and a programme for parents) and Adolescent Watch (comparison group: normal school curriculum) ${ }^{28}$ Both intervention groups (RAP-A and RAP-F) had a significantly greater decrease in depressive symptoms at post intervention and 10-month follow-up, compared with the comparison group. Another trial, in Auckland, New Zealand, compared RAP-Kiwi (the New Zealand version of RAP) with a placebo. ${ }^{29}$ In both study arms, participants completed 11 sessions during school hours. The placebo programme focused on fun, art-and-craft-style activities, without the CBT aspects thought to actively prevent depression. Post intervention, depression scores were reduced significantly more in the RAP-Kiwi group than the placebo group, for two depression measures (the Reynolds Adolescent Depression Scale and Beck Depression Inventory II). At 18 months post intervention, the Reynolds Adolescent Depression Scale scores were significantly different between groups. The RAP has also been trialled in secondary schools in Mauritius, where one group received RAP-A and the other group was assigned to a waitlist as the comparison group. ${ }^{30}$ This trial found that in comparison with the control group, the intervention group had significant changes in self-esteem and coping skills at post intervention and six-month follow-up, and decreased symptoms of depression immediately post intervention, but not at six-month follow-up.

Most LALMIC governments have very limited national policies and programmes for child and adolescent mental health due to financial constraints and other social and health priorities. In the WHO Atlas Project, which reviewed services, training and policy for child and adolescent mental health worldwide, only a quarter of LALMICs had any national mental health policies and none had programmes for child and adolescent mental health. $^{2}$ Recent systematic reviews of interventions for adolescent mental health found that only 4/38 studies, and less than $0.1 \%$ of global mental health research relevant to adolescents, were conducted in a LMIC. ${ }^{321}$

In Vietnam, mental health, including that of adolescents, is poorly recognised. Advances in recognition of mental health and the importance of appropriate care have been made, especially with the Prime Minister of Vietnam's legislated Decision 1215/QD-TTg in 2011. ${ }^{31}$ This policy acknowledges the importance of mental health and supports enhanced community care services for people with mental illness. Several school-based mental healthcare programmes have been implemented and evaluated. ${ }^{32}$ However, these were limited to small, provincial-focused programmes and none have been shown to be effective. Despite these advances, recognition of mental health problems is still restricted to severe psychiatric conditions, with common mental disorders, including depression and anxiety, not adequately considered. ${ }^{33}$ Stigma associated with mental health problems remains high, and is a major barrier to improving mental health in Vietnam. ${ }^{34}$

\section{Objectives}

The aim of this study is to translate, culturally verify and adapt the RAP for Vietnamese adolescents, combined with the strength-focused psychoeducational resources for teachers (Happy House); and establish the effectiveness 
of the Happy House intervention in improving the mental health of adolescents in Vietnam. We hypothesise that the adapted resilience intervention will reduce symptoms of depression, anxiety and health risk behaviours; and increase coping self-efficacy, anger management and school connectedness among adolescents in Vietnam.

\section{METHODS AND ANALYSIS RAP adaptation process}

The adaptation process will include several steps: (1) consultation process with experts in Australia and Vietnam, and with school teachers and adolescents in Vietnam about need for an feasibility of conducting a school-based programme; (2) translation of the RAP materials into Vietnamese and linguistic and cultural adaptation for the local context; (3) back translation into English and review of the translated version and (4) a pilot test of the Vietnamese version of RAP (Happy House) in a high school in Vietnam.

\section{Consultation with local and international experts}

The initial consultation with bilingual Vietnamese psychologists and psychiatrists who have expertise in adolescent mental health, high school teachers and grade 10 students will be conducted to identify the preliminary acceptability and feasibility of RAP for use in Vietnam. The RAP materials in English will be sent to the stakeholders in advance. The research team will conduct faceto-face interviews and group discussions to obtain their opinions about whether the content, metaphors, format and illustrated videos are appropriate for Vietnamese adolescents, and their suggestions for changes.

All comments and suggested changes will be discussed among the Vietnamese and Australian research team members, including the authors of RAP, to make a revised version of the intervention in English.

\section{Translation and cultural adaptation}

The revised RAP will be translated into Vietnamese by Vietnamese researchers who are bilingual psychologists. The Vietnamese version of RAP will be called 'Happy House'. Happy House will be back translated by an independent professional translator into English. The RAP authors will review the back translated version of Happy House and work with the Vietnam team to make any changes needed. Based on the revised English version, Happy House in Vietnamese will be revised to create a second draft.

\section{Pilot testing}

Key sessions of the second draft version of Happy House will be piloted among a group of 10-15 grade 10 students in a high school in Hanoi. A group discussion will be conducted after each session to obtain comments and suggestions. All necessary changes to Happy House will be discussed to reach a consensus among the research team, to make the final version of Happy House.

\section{Trial design}

This is a two-arm parallel controlled trial of Happy House. Students in grade 10 from randomly selected classes in selected high schools in the north of Vietnam will be invited to participate. In the intervention arm, students will receive six weekly $90 \mathrm{~min}$ school-based group sessions. Students will receive text messages between the sessions (two times per week) via a social network (Zalo/Facebook messenger). The text messages will be short (under 60 characters), include graphics and remind students about key messages of each session. In the control arm, students and teachers will receive only the usual curriculum, which does not include any mental health programmes.

\section{Study setting}

Vietnam is a rapidly developing lower-middle income Southeast Asian country. Most (65.6\%) of the country's 96 million people live in rural areas and rely on subsistence agriculture. $^{35}$ The average national per capita income in 2018 was US $\$ 2360$, with major disparities between urban and rural areas ${ }^{36}$ Children and adolescents account for a third of the population and are often required to participate in income-generating activities outside school hours, especially in rural areas. Hanoi is the capital city, with a population of 7 million people, residing in 12 urban districts and 18 rural provinces.

\section{Participants}

Eligibility criteria

All students in grade 10 (usually aged 15-16 years) studying in selected classes.

\section{Exclusion criteria}

Students whose parents do not give permission for them to participate, or students who do not wish to participate, will be excluded from the study.

\section{Intervention}

Happy House will consist of materials incorporating the principles of RAP, which include workshop-style exercises for adolescents focusing on the six core components of RAP: personal strengths, managing stress, cognitive style, problem solving, support networks and interpersonal relationships. Key messages of Happy House will be sent by text message to students between sessions to reinforce content learnt in the Happy House sessions.

The programme will be delivered within school hours through sixweekly $90 \mathrm{~min}$ sessions in groups of 15-20 students in their classrooms by Vietnamese research assistants and class teachers (core facilitators). The Vietnamese research assistants and class teachers will be trained by the RAP authors. The research team will provide supportive supervision to the core facilitators, with technical advice from the RAP authors.

\section{Outcomes}

Primary and secondary outcomes of the resilience process of adaptation in the context of adversity are described in table 1. These include the changes in markers of 
Table 1 Data sources for assessment of outcomes

\begin{tabular}{|c|c|c|}
\hline Variable & Measurement & Description \\
\hline \multicolumn{3}{|l|}{ Primary outcome } \\
\hline Depression & $\begin{array}{l}\text { Centre for Epidemiologic Studies } \\
\text { Depression Scale Revised }\end{array}$ & $\begin{array}{l}20 \text {-item scale, assessing depressive symptoms. Has been } \\
\text { validated for use among adolescents in Vietnam. }{ }^{40}\end{array}$ \\
\hline \multicolumn{3}{|l|}{ Secondary Outcomes } \\
\hline Mental well-being & $\begin{array}{l}\text { Mental Health Continuum Short } \\
\text { Form }^{4142}\end{array}$ & $\begin{array}{l}\text { 14-item scale representing three dimensions of well-being: } \\
\text { emotional, social and psychological well-being. }\end{array}$ \\
\hline School connectedness & $\begin{array}{l}\text { School connectedness scale } \\
\text { developed by The National } \\
\text { Longitudinal Study of Adolescent } \\
\text { Health }^{44}\end{array}$ & $\begin{array}{l}\text { This scale measures the bond adolescents' feel toward } \\
\text { school through the responses to five statements: 'I feel } \\
\text { close to people at this school', 'I feel like I am part of this } \\
\text { school', 'I am happy to be at this school', 'The teachers } \\
\text { at this school treat students fairly' and 'I feel safe in my } \\
\text { school'. }\end{array}$ \\
\hline Health risk behaviours & $\begin{array}{l}14 \text { questions selected from the Youth } \\
\text { Risk Behavior Survey } \\
\text { questions }{ }^{46}\end{array}$ & $\begin{array}{l}\text { Health behaviours are assessed including cigarette } \\
\text { smoking, alcohol use, physical fighting, watching TV, } \\
\text { playing video games and using a computer. }\end{array}$ \\
\hline
\end{tabular}

adaptation, such as mental health and well-being and the protective factors such as coping self-efficacy and anger management.

\section{Baseline characteristics}

Sociodemographic characteristics: study-specific questions will be used to assess age, sex, number of household members, and parents' education and occupation.

Physical health: having a chronic disease or disability will be assessed by study-specific questions.

Burden of academic activities: educational stress, including pressure from study, worry about grades, despondency, self-expectation and workload will be assessed using the Educational Stress Scale for Adolescents. ${ }^{37}$

\section{Participant timeline}

In both study arms, baseline data (B) will be collected one week after recruitment. There will be two follow-ups: within twoweeks post intervention (F1), and after six months (F2). Table 2 shows the detailed time schedule of enrolment, interventions and assessments.

\section{Sample size calculation}

The number of clusters and sample size has been calculated using the sampsi command in Stata, V.16. A total of 1004 adolescents (each study arm: 502 adolescents) is needed to detect a difference in the primary outcome (Centre for Epidemiologic Studies Depression Scale Revised Score $\geq 16$ ) of $41 \%$ in the control arm and $31 \%$ in the Happy House intervention arm (power of $90 \%$, significance level of 0.05 ). These assumptions are based on previous studies in Vietnam. ${ }^{131618}$ Taking into account a refusal rate of $10 \%$ and an attrition rate of $10 \%$, a total of 1204 adolescents will be invited to participate.

\section{Recruitment and assignment of interventions}

Two from a total of twelve urban districts and two from a total of eighteen rural districts in Hanoi will be selected randomly. In each district, two high schools will be randomly selected, and one randomly assigned to the intervention and the other to the control arm. Randomisation will be conducted using sequences of computer-generated random numbers, with assistance from Stata V.16.0. This selection process will be carried out by an independent statistician. In each of the selected schools, four grade 10 classes will be chosen randomly.

All students in the selected classes will be invited to participate. At this year level, there are 30-45 students in each class. An information package, which will consist of two explanatory statements (one for the student and the other for their parent/s or guardian/s) and a consent form, will be delivered to each student in the selected classes several days before the administration of the baseline survey. Students will be advised to give the explanatory statement and consent form to their parent/s or guardian/s. It will be clearly explained that participation is voluntary and whether or not students participate will not affect their schooling in any way. Students will be asked to obtain written consent from one parent or guardian and return it to the data collectors on the day of the survey administration. It will be emphasised that any questions about the project can be answered by contacting the researchers. The researchers' contact details will be provided in the explanatory statements and students will be reminded of these during the distribution of the information packages. Only students who wish to participate and whose parent/guardian give written 
Table 2 Participant timeline

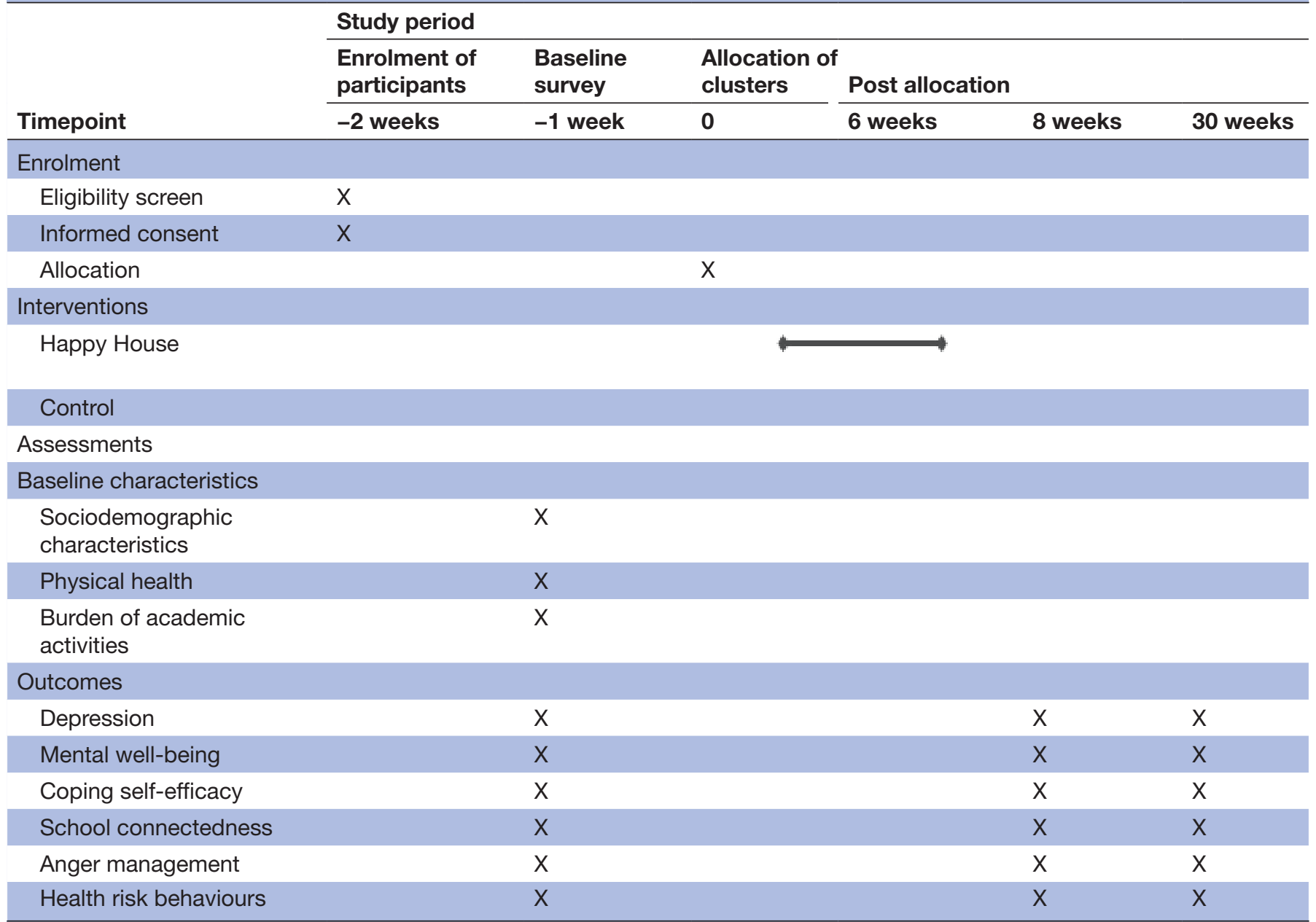

consent for participation will be eligible to participate in this study.

\section{Data collection procedure}

Baseline and follow-up assessments with the students will be conducted using self-completed questionnaires during a usual $45 \mathrm{~min}$ class session, monitored and supervised by two trained data collectors from the Hanoi University of Public Health (HUPH). Students will be given instructions on how to complete the questionnaire and asked to return the completed questionnaire sealed in an envelope, which will be given to them at the beginning of the session. The administration of the survey will be conducted in the presence of the data collectors only, without any teaching or intervention staff present.

The students who do not want to participate and students whose parent/guardian does not grant consent for them to participate will be invited to the school library to do their homework.

\section{Blinding}

Data collectors and data analysts will be blinded to the assignment of schools to trial arms. Participants will each be assigned a code number which identifies the school, but not the trial arm. Trial assignment of each school will only be made available to data analysts after unblinding at the end of the trial.

\section{Plans to promote participant retention}

Participation rates of each class assigned to the intervention arm for each session will be closely monitored using facilitator reports and attendance sheets. If the participation rate falls below $70 \%$, it will be followed up and participants in the group will be visited by a senior facilitator to identify any difficulties or obstacles that prevent participation. Barriers will be addressed where possible. However, participation is totally voluntary.

\section{Data management}

Data from the paper-based questionnaires will be entered into a customised Microsoft Access database by trained data entry officers of HUPH. Data will be checked and cleaned by a data manager at HUPH.

\section{Statistical methods}

Descriptive analyses will be used to describe participants' characteristics. Differences in the primary and secondary outcomes between the two arms will then be estimated by individual-level analyses adjusting for clustering. Mixed-effect logistic regression for the main outcome and mixed-effect 
linear and logistic regression models for the secondary outcomes, taking into account covariates and prognostic factors (such as age, sex, rural/urban residence, socioeconomic status and baseline data of the outcomes) and incorporating random effects for school and class and a fixed effect for trial arm will be conducted to estimate the effects of the intervention on the outcomes. All analyses will follow intention-to-treat principles, applying multiple imputations for missing data and be performed at individual level using Stata, V.16 (StataCorp LP, College Station, Texas, USA). Attendance at the weekly sessions (comparing outcomes between those who participated or did not) will be used in a sensitivity analysis. Finally, mediation analyses will be conducted using the multilevel structural equation modelling technique in MPlus V.8 (Muthén and Muthén, Los Angeles, California, USA). The mediators that will be tested are the secondary outcomes (table 1), as they are the intermediate outcomes that this intervention targets to change.

\section{Data monitoring}

Data monitoring will be conducted by an independent Data Monitoring Committee (DMC), including a biostatistician, a paediatrician and a public health physician. The DMC will follow the Australian National Health and Medical Research Council guidelines for Data Safety Monitoring Boards. ${ }^{38}$ The DMC will assess the safety data and overall progress of the trial at the middle of intervention (threeweeks after the start) and the outcome data at twoweeks post intervention. Depending on the results, the DMC will provide recommendations to the research team whether to continue, modify or stop the trial.

\section{Harm}

In case of participant distress, contact details of supporting services for children and adolescents in Vietnam, including the free helpline, local police and Youth Union will be provided. Referrals to appropriate supporting services will be made for those who feel distressed during the intervention and need access to these services.

\section{Patient and public involvement}

Adolescents and policy makers will participate in the adaptation process of the RAP.

\section{Quality control}

Effort will be made to control the quality of this trial in several ways. The intervention will be delivered by a group of core facilitators, made up of Vietnamese research assistants and teachers. These core facilitators will be trained by the RAP authors to ensure that they are taught the RAP principles and methods accurately. The programme will be trialled in a pilot format with a small group of students, to ensure that it is appropriate and understandable, and changes may be made in response to this feedback. As described above, an independent DMC will monitor the quality, progress and safety of the trial, and can make recommendations to continue, modify or stop the trial if necessary.

\section{ETHICS AND DISSEMINATION}

\section{Research ethics approval}

The conduct of the trial has been approved by Monash University Human Research Ethics Committee (Approval Number: 21455), Melbourne, Victoria, Australia and the Institutional Review Board of the Hanoi School of Public Health (Certificate Number: 488/2019/YTCC-HD3), Hanoi, Vietnam.

\section{Protocol amendments}

All modifications to the protocol will be reported to the investigators through regular meetings or emails, ethics committees through amendment application forms or by email if these are not used, trial participants through announcements on commune notice boards, and trial registries through the registration portal. The chief investigator with the project manager will take responsibility for ensuring that these are completed.

\section{Consent}

Students' and their parent's/guardian's written consent will be sought. The researchers will explain to students that participation is voluntary, and that non-participation will not affect their schooling in any way.

\section{Confidentiality}

Participants' confidentiality will be maintained at all times. All data will be coded and deidentified. All the collected data and consent forms with participant identifiers will be securely stored at the HUPH and Monash University. Electronic data will be stored in electronic format on a password protected folder on the shared drive at the HUPH and Monash University. All the data and forms will be kept for a minimum period of 5 years after final publication in a secure location in accordance with Monash University Intellectual Property Right Policies. Only the researchers will have access to stored information. All identifiable data will be destroyed permanently after 5 years.

\section{Access to data}

Deidentified individual data will be made available to the public at https://monash.figshare.com from December 31 2021.

\section{Dissemination policy}

We will use a multistranded dissemination strategy to ensure translation of findings. Dissemination will include peer-reviewed publications, international and national conferences, seminar and media presentations, national policy briefings in Vietnam, local language reports and lay language summaries for schools and participants.

\section{DISCUSSION}

This study will provide essential evidence for sustainable integration of a cultural and linguistical adaptation of the RAP into the existing school curriculum to reduce prevalent adolescent mental health problems and to promote positive mental health in Vietnam. Better mental health 
and well-being will assist adolescents to reach their optimal development and will increase the likelihood of good mental health and well-being in adulthood. The programme may contribute to the reduction of burden of disability due to mental health problems and associated costs to society. To promote its sustainability, the culturally adapted RAP may be taken up by Vietnam's Ministry of Education and Training and incorporated into the curriculum for high school students. These are not limited to adolescents in Vietnam, but also adolescents living in other LALMICs. If proven effective, this programme can be considered to be adapted and used widely in other resource constrained settings.

\section{Trial status}

Trial recruitment will commence in October 2020 and, at the time of manuscript submission, has not commenced.

Contributors $\mathrm{JF}, \mathrm{TT}$ and $\mathrm{ML}$ designed the study and prepared the first draft. RS and TN contributed to preparing the first draft. HTN, IS, AW, JO, NN, NL and HN contributed to the design of the study and reviewed and contributed to revisions of the manuscript. All authors agreed on the content of the final submitted version.

Funding This research is funded by Australian National Health and Medical Research Council—NAFOSTED Joint Call for Collaborative Research Projects (GNT1158429). TT is supported by an Australian National Health and Medical Research Council Early Career Research Fellowship and a Monash Strategic Bridging Fellowship. JF is supported by the Finkel Professorial Fellowship, which is funded by the Finkel Family Foundation.

\section{Competing interests None declared.}

Patient and public involvement Patients and/or the public were involved in the design, or conduct, or reporting, or dissemination plans of this research. Refer to the Methods and analysis section for further details.

Patient consent for publication Not required.

Provenance and peer review Not commissioned; externally peer reviewed.

Open access This is an open access article distributed in accordance with the Creative Commons Attribution Non Commercial (CC BY-NC 4.0) license, which permits others to distribute, remix, adapt, build upon this work non-commercially, and license their derivative works on different terms, provided the original work is properly cited, appropriate credit is given, any changes made indicated, and the use is non-commercial. See: http://creativecommons.org/licenses/by-nc/4.0/.

\section{ORCID iDs}

Thach Tran http://orcid.org/0000-0002-4686-8601

lan Shochet http://orcid.org/0000-0002-4666-2128

Jane Fisher http://orcid.org/0000-0002-1959-6807

\section{REFERENCES}

1 Fisher J, Cabral de Mello M, Izutsu T, et al. Adolescent mental health in resource-constrained settings: a review of the evidence of the nature, prevalence and determinants of common mental health problems and their management in primary health care. Int $J$ Soc Psychiatry 2011;57:9-116.

2 Belfer ML. Child and adolescent mental disorders: the magnitude of the problem across the globe. J Child Psychol Psychiatry 2008;49:226-36.

3 Klasen $\mathrm{H}$, Crombag A-C. What works where? A systematic review of child and adolescent mental health interventions for low and middle income countries. Soc Psychiatry Psychiatr Epidemiol 2013;48:595-611.

4 Bronfenbrenner U. The ecology of human development: experiments by nature and design. Cambridge, Mass: Havard University Press, 1979.

5 Patel V, Flisher AJ, Hetrick S, et al. Mental health of young people: a global public-health challenge. Lancet 2007;369:1302-13.

6 Amstadter AB, Richardson L, Meyer A, et al. Prevalence and correlates of probable adolescent mental health problems reported by parents in Vietnam. Soc Psychiatry Psychiatr Epidemiol 2011;46:95-100.

7 Knapp M. Schizophrenia costs and treatment cost-effectiveness. Acta Psychiatr Scand Supp/ 2000;407:15-18.

8 Leibson CL, Katusic SK, Barbaresi WJ, et al. Use and costs of medical care for children and adolescents with and without attentiondeficit/hyperactivity disorder. JAMA 2001;285:60-6.

9 Le MTH, Holton S, Romero L, et al. Polyvictimization among children and adolescents in low- and Lower-Middle-Income countries: a systematic review and meta-analysis. Trauma Violence Abuse 2018;19:323-42.

10 Luthar SS, Cicchetti D, Becker B. The construct of resilience: a critical evaluation and guidelines for future work. Child Dev 2000;71:543-62.

11 Betancourt TS, Khan KT. The mental health of children affected by armed conflict: protective processes and pathways to resilience. Int Rev Psychiatry 2008;20:317-28.

12 Shochet I, Dadds M. Adolescent depression and the family: a paradox. Clin Child Psychol Psychiatry 1997;2:307-12.

13 Le MTH, Holton S, Nguyen HT, et al. Poly-victimisation and health risk behaviours, symptoms of mental health problems and suicidal thoughts and plans among adolescents in Vietnam. Int $J$ Ment Health Syst 2016;10:66

14 Le MTH, Holton S, Nguyen HT, et al. Poly-Victimisation among Vietnamese high school students: prevalence and demographic correlates. PLoS One 2015;10:e0125189.

15 Le MTH, Holton S, Nguyen HT, et al. Victimisation, poly-victimisation and health-related quality of life among high school students in Vietnam: a cross-sectional survey. Health Qual Life Outcomes 2016;14:155

16 Nguyen HT, Dunne MP, Le AV. Multiple types of child maltreatment and adolescent mental health in Viet Nam. Bull World Health Organ 2010;88:22-30.

17 Akmatov MK. Child abuse in 28 developing and transitional countries--results from the Multiple Indicator Cluster Surveys. Int J Epidemiol 2011;40:219-27.

18 Nguyen DT, Dedding C, Pham TT, et al. Depression, anxiety, and suicidal ideation among Vietnamese secondary school students and proposed solutions: a cross-sectional study. BMC Public Health 2013;13:1195.

19 MT L, Holton S, Nguyen HT, et al. "I feel that life is meaningless": Vietnamese adolescents' experiences of and reflections about interpersonal violence. Glob Ment Health 2018;5.

20 Phuong TB, Huong NT, Tien TQ, et al. Factors associated with health risk behavior among school children in urban Vietnam. Glob Health Action 2013;6:18876-9.

21 Das JK, Salam RA, Lassi ZS, et al. Interventions for adolescent mental health: an overview of systematic reviews. J Adolesc Health 2016:59:S49-60.

22 Oveisi S, Ardabili HE, Dadds MR, et al. Primary prevention of parentchild conflict and abuse in Iranian mothers: a randomized-controlled trial. Child Abuse Negl 2010;34:206-13.

23 Cowan FM, Pascoe SJS, Langhaug LF, et al. The Regai Dzive Shiri project: results of a randomized trial of an HIV prevention intervention for youth. AIDS 2010;24:2541-52.

24 Shochet IM, Wurfl A. Resourceful Adolescent Program: Group Leader's Manual. Brisbane, Australia: School of Psychology and Counselling, Queensland University of Technology, 2015.

25 Beck AT, Rush AJ, Shaw BF, et al. Cognitive therapy of depression: a treatment manual. New York, NY: Guilford Press, 1979.

26 Mufson L, Moreau D, Weissman MM, et al. Interpersonal psychotherapy for adolescent depression. In: Klerman GL, Weissman MM, eds. New applications of interpersonal psychotherapy. Washington, DC: American Psychiatric Association Press, 1993: 130-66.

27 Zhou X, Hetrick SE, Cuijpers P, et al. Comparative efficacy and acceptability of psychotherapies for depression in children and adolescents: a systematic review and network meta-analysis. World Psychiatry 2015;14:207-22.

28 Shochet IM, Dadds MR, Holland D, et al. The efficacy of a universal school-based program to prevent adolescent depression. J Clin Child Psychol 2001;30:303-15.

29 Merry S, McDowell H, Wild CJ, et al. A randomized placebocontrolled trial of a school-based depression prevention program. $J$ Am Acad Child Adolesc Psychiatry 2004;43:538-47.

30 Rivet-Duval E, Heriot S, Hunt C. Preventing adolescent depression in Mauritius: a universal school-based program. Child Adolesc Ment Health 2011;16:86-91.

31 The Prime Minister. Approving the project on social support and functional recovery for psychiatric and mental disorder patients based on the community for the period 2011-2020. Hanoi, 2011. 
32 Bệnh viện tâm thần ban ng y Mai Hương. Chương trình chăm sóc sức khỏe tâm thần học đường. secondary Chương trình chăm sóc sức khỏe tâm thần học đường, 2014. Available: https://www.maihuong. gov.vn/vi/quan-he-quoc-te/91-chuong-trinh-suc-khoe-tam-than-hocduong-.html

33 MTH L. Poly-victimisation and mental health and quality of life of adolescents in Vietnam. Monash University, 2017.

34 Minas H, Edington C, La N, et al. Mental health in Vietnam. In: Minas HLM, ed. Mental health in Asia and the Pacific. Boston, MA: Springer, 2017: 145-61.

35 Central Population and Housing Census Steering Committee. The Vietnam population and housing census of 00:00 hours on 1 April 2019. Hanoi: General Statistics Office of Viet Nam, 2019.

36 World Bank. Gdp (current USS). secondary GDP (current USS), 2017. Available: https://data.worldbank.org/indicator/NY.GDP.MKTP.CD? locations $=A U-V N \&$ view $=$ chart

37 Sun J, Dunne MP, Hou X-Y, et al. Educational stress scale for adolescents: development, validity, and reliability with Chinese students. J Psychoeduc Assess 2011;29:534-46.

38 National Health and Medical Research Council. Data safety monitoring boards (DSMBs). Canberra: Australian Government, 2018.

39 Eaton WW, Muntaner C, Smith C, et al. Center for Epidemiologic Studies Depression Scale: Review and revision (CESD and CESD-R).
In: Maruish ME, ed. The use of psychological testing for treatment planning and outcomes assessment. 3rd ed.. Mahwah, NJ: Lawrence Erlbaum, 2004: 363-77.

40 Nguyen T, Le V, Dunne M. Validity and reliability of depression and anxiety scales using in community-based adolescent research. Viet $J$ Pub Health 2007;7:25-31.

41 Keyes CLM, Wissing M, Potgieter JP, et al. Evaluation of the mental health continuum-short form (MHC-SF) in setswana-speaking South Africans. Clin Psychol Psychother 2008;15:181-92.

42 Keyes CL. The subjective well-being of America's youth: Toward a comprehensive assessment. Adolescent \& Family Health, 2006.

43 Chesney MA, Neilands TB, Chambers DB, et al. A validity and reliability study of the coping self-efficacy scale. Br J Health Psychol 2006;11:421-37.

44 Sieving RE, Beuhring T, Resnick MD, et al. Development of adolescent self-report measures from the National longitudinal study of adolescent health. $J$ Adolesc Health 2001;28:73-81.

45 Linden W, Hogan BE, Rutledge T, et al. There is more to anger coping than "in" or "out". Emotion 2003;3:12-29.

46 Centers for Disease Control and Prevention. 2019 youth risk behavior survey questionnaire. secondary 2019 youth risk behavior survey questionnaire. Available: www.cdc.gov/yrbs 